\title{
Télécommunications Numériques sous Labview
}

\author{
Frédéric Bonnardot \\ frederic.bonnardot@univ-st-etienne.fr \\ Université de Lyon - Université Jean Monnet - IUT Réseau et Télécoms de Roanne - LASPI \\ Laboratoire d'Analyse des Signaux et des Processus Industriels \\ IUT Réseau et Télécoms de Roanne 20, Avenue de Paris 42334 Roanne Cedex
}

\begin{abstract}
RESUME : Afin d'étudier les problèmes liés à la synchronisation en télécommunication numérique et de construire des TP avec peu de matériel, un système de télécommunication basé sur une carte son, un microphone, ainsi que le logiciel Labview a été développé à l'IUT de Roanne. Cette communication présente dans une première partie le public visé, puis une deuxième partie montre brièvement les outils mis à disposition des étudiants, leurs intérêts didactiques (temps réel, signaux audibles, ...). Une dernière partie traite de l'approche adoptée afin de montrer aux étudiants les problèmes rencontrés lors de la réalisation d'un émetteur - récepteur numérique et surtout montre comment les faire réfléchir à des solutions simples permettant de les résoudre.
\end{abstract}

Mots clés : télécommunication numérique, Labview, BPSK, IUT.

\section{INTRODUCTION}

Suite à la création récente (2006) d'un département réseau et télécommunications (R\&T) à l'IUT de Roanne, des modules télécommunications ont dû être créés sans utiliser le matériel classique (analyseur de spectre vectoriel, ...).

Afin de rendre d'une part les TP plus attractifs pour les étudiants et d'autre part d'utiliser du matériel rapidement disponible, les TP ont été créés à l'aide d'enceintes audio et de microphones. Les étudiants ont dû mettre en place une chaîne de transmission numérique en assemblant des modules préparés par l'enseignant sous Labview (langage de programmation graphique). Les étudiants découvraient au fur et à mesure les problèmes de la télécommunication numérique (synchronisation, récupération de rythme, ...) et recherchaient des solutions.

La première partie présente le contexte pédagogique, la deuxième partie le matériel et les logiciels utilisés pour ce TP, enfin la troisième partie montre les outils mis à disposition aux étudiants et la dernière partie indique comment ces outils sont exploités lors du TP.

\section{PRÉSENTATION DU CONTEXTE}

Le DUT R\&T est une formation dans le domaine des télécommunications, des réseaux et de l'informatique. Parmi les débouchés de cette formation, on trouve :

- administrateur de réseaux informatiques,

- informaticiens WEB,

- consultant en réseaux d'entreprise,

- technicien en téléphonie mobile et réseaux publics,

- technico-commercial en réseaux ou en téléphonie d'entreprise, ...

Les étudiants sont issus aussi bien de baccalauréats $\mathrm{S}$ que STI et professionnels. Étant donné les spécialités enseignées, le niveau mathématique est plus relevé que les IUT classiques. Dès lors, il devient indispensable de fournir un effort pour rendre les cours et TP attrayants et accessibles pour les étudiants.

Le TP présenté ici intervient en début de deuxième année dans le module de télécommunications numériques (TR1). Le but de ce module est d'étudier les notions de codage source, codage canal, et, les techniques de communications numériques classiques (BPSK, QAM, ...) et avancées (OFDM, CDMA).

Le but de ce TP réparti sur 3 séances de 4 heures est d'amener les étudiants à réaliser un émetteur / récepteur numérique et de comprendre les problèmes de synchronisation lors de la réception et d'introduire le module E4 traitant des systèmes bouclés appliqués aux télécommunications.

Les techniques avancées telles que l'OFDM sont vues en analysant des signaux de type wifi à l'aide d'analyseurs de spectre vectoriels.

\section{PRÉSENTATION DE LA MAQUETTE}

\subsection{Le matériel}

Le matériel nécessaire est constitué d'un microphone, d'une paire d'enceintes (intégrées aux moniteurs), et d'un PC.

Il faut choisir un microphone et une enceinte bas de gamme. En effet, ils servent à simuler l'antenne et une partie des dégradations provoquées en pratique par le canal de propagation.

Le PC doit être rapide (éviter les celeron), car les traitements sont effectués en temps réel. L'aspect temps réel, bien que non nécessaire, est plus attrayant pour les étudiants. 


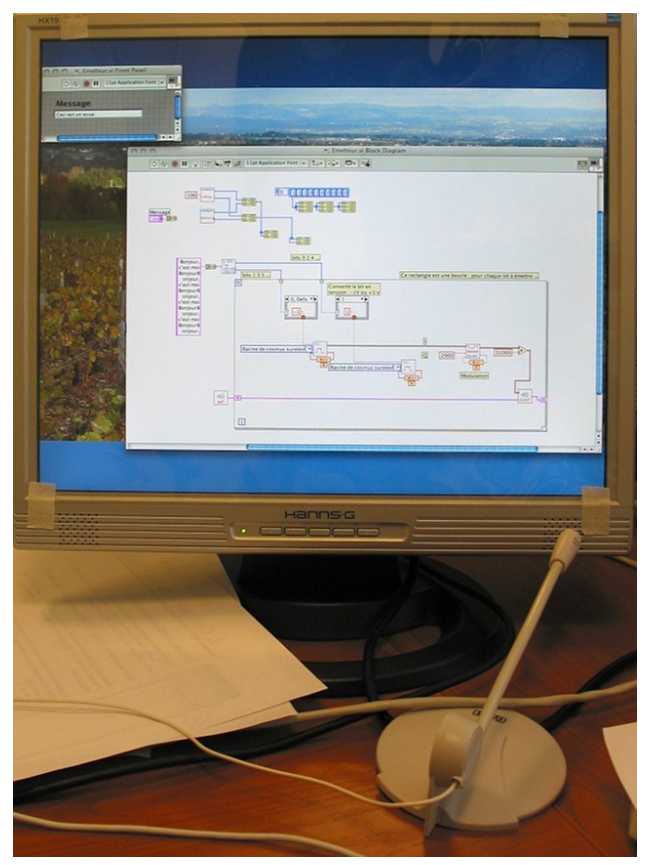

fig 1 : Matériel utilisé : enceintes de l'écran et microphone

\subsection{Les logiciels}

Les traitements sont effectués sous Labview. Labview est un langage de programmation graphique (comme Simulink, Mustig [1], SciOs [2], ...). Il permet de décrire le fonctionnement du modulateur / démodulateur d'une manière proche d'un schéma fonctionnel (fig. 2).

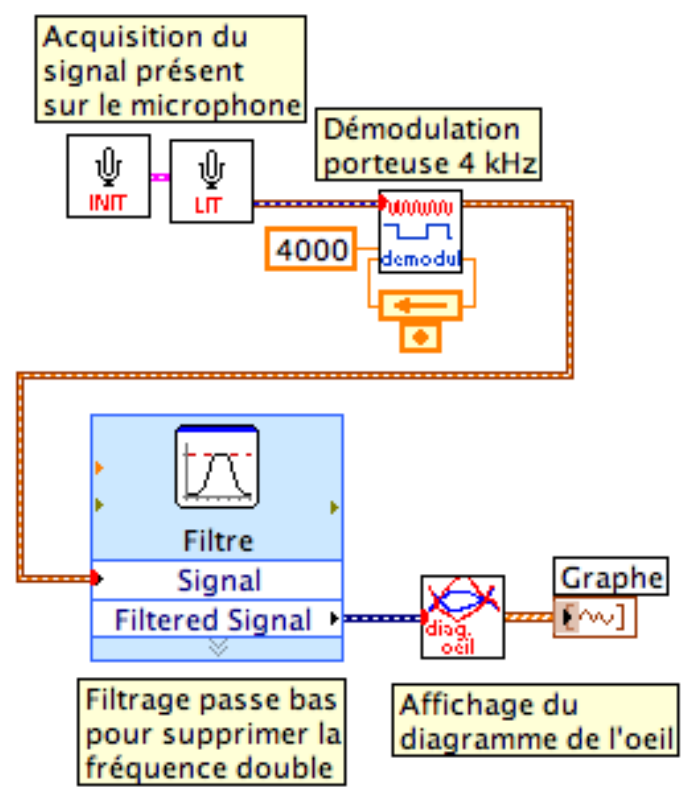

fig 2 : Exemple de diagramme Labview

\section{DESCRIPTION DE LA BOITE À OUTILS}

Les fonctions fournies par la boîte à outils télécommunication de Labview étant trop exhaustives et trop complexes pour des étudiants de niveau IUT, une boîte à outils dédiée a été développée. Dès lors, il devient possible, en tant que concepteur, de masquer certain aspect aux étudiants pour ce concentrer sur l'essentiel.

La boîte à outils contient un ensemble de fonctions (par exemple, les blocs INIT, LIT, ... visibles sur la fig. 2) ainsi qu'un fichier contenant des constantes. Ce dernier permet de limiter le nombre de paramètres associés aux fonctions (et donc le nombre de fils).

\subsection{Constantes}

Les constantes sont :

- la fréquence d'échantillonnage,

- le nombre d'échantillons associé à un symbole,

- ainsi que le nombre de symboles Nsymb traité simultanément en réception.

Les étudiants n'ont pas à modifier ces constantes durant le TP.

\subsection{Gestion de la carte son}

La carte son est tout d'abord initialisée, puis les signaux sont transférés à la carte son par l'intermédiaire d'un bloc dédié à l'acquisition du signal présent à la sortie du microphone et d'un bloc dédié à l'envoi d'un signal au convertisseur associé aux enceintes.

Étant donné que la fréquence d'échantillonnage est stockée dans le fichier constante, le seul paramètre associé à ces blocs est le signal acquis pour LIT et le signal à écouter pour le bloc ECRIT.

Les données ne sont pas traitées point par point, mais par bloc d'un symbole pour l'émission et par bloc d'environ $\mathrm{Nsymb}=10$ symboles pour la réception. La fréquence d'échantillonnage est volontairement basse $(8 \mathrm{kHz})$ afin de permettre d'afficher plusieurs graphes en temps réel et d'être plus réactif.

Les parties émission et de réception sont volontairement indépendante afin d'introduire des erreurs de phase. Ce déphasage lié à l'instant de démarrage de l'émetteur oblige l'étudiant à écrire un algorithme pour déterminer l'instant de décision optimum qui change à chaque démarrage de l'émetteur.

L'utilisation de bloc de 10 symboles en réception permet de calculer facilement un diagramme de l'oeil en découpant ces 10 symboles en tranches d'un symbole qui seront ensuite superposées. 


\subsection{Message et génération de symbole}

Le message est constitué par d'un texte transformé en deux trains de bit à l'aide d'un bloc fourni (voir fig. 3). Les étudiants disposent de bloc permettant la mise en forme du signal. Ils peuvent choisir entre une mise en forme de type rectangulaire ou racine de cosinus surélevé). Il est également possible (non présent sur la fig. 3) d'utiliser un bloc fournissant en sortie deux séquences de «démarrage » (une pour la voie I et une pour la voie Q).

Pour le récepteur, un filtre adapté (rectangle ou racine de nyquist) ainsi que des outils permettant de reconstituer le message sont fournis.

La partie réception est plus complexe, car elle nécessite une synchronisation symbole, une synchronisation de porteuse, et la détermination de l'instant de décision. Tous ces éléments correspondent à des blocs qui sont placés par les étudiants au fur et à mesure que le TP avance et que l'on se rapproche des conditions réelles.

Pour ces outils, les étudiants mettent en évidence un problème, recherchent avec l'aide de l'encadrant une solution à ce problème et utilisent ensuite le bloc correspondant à la solution envisagée. Cette méthode permet de faire réfléchir les étudiants sans perdre de temps en programmation.

Des outils de détection de la séquence de démarrage, d'échantillonnage, ainsi que de démultiplexage sont également fournis.

\subsection{Modulation}

Les étudiants disposent d'un bloc pour la modulation et la démodulation. Il est possible d'agir sur la phase pour synchroniser l'émetteur et le récepteur. La fréquence porteuse est également réglable (en émission et réception).

\subsection{Outils d'analyse}

En plus des graphes, des blocs permettant de construire facilement un diagramme ou une constellation sont fournis.

Le paramétrage des blocs a été volontairement limité au maximum (niveau IUT). Parmi les paramètres accessibles aux étudiants figurent : la fréquence porteuse, le type de mise en forme, la fréquence des filtres passe-bas, la phase, ...

\section{EXPLOITATION}

Le TP réalisé sur 3 séances ce compose de plusieurs phases :

- réalisation de l'émetteur où les étudiants se familiarisent avec les blocs, l'utilisation de Labview, et, leurs cours de télécommunication (4 heures),

- $\quad$ artie démodulation et filtrage adapté avec étude des problèmes de phase (4 heures),

- échantillonnage du symbole, prise de décision et reconstruction du message (4 heures).

Nous ne décrirons pas ici les aspects techniques de programmation Labview (le TP pouvant être réalisé avec d'autres outils du même type), mais nous nous focaliserons plutôt sur les questions posées aux étudiants et leurs démarches.

\subsection{Réalisation de l'émetteur (4 heures)}

Pour réaliser l'émetteur (fig. 3), les étudiants :

- tapent un texte,

- le transforment en train de bit,

- associent chaque bit à une tension,

- mettent en forme l'impulsion associée,

- la modulent,

- envoient le résultat sur la carte son.

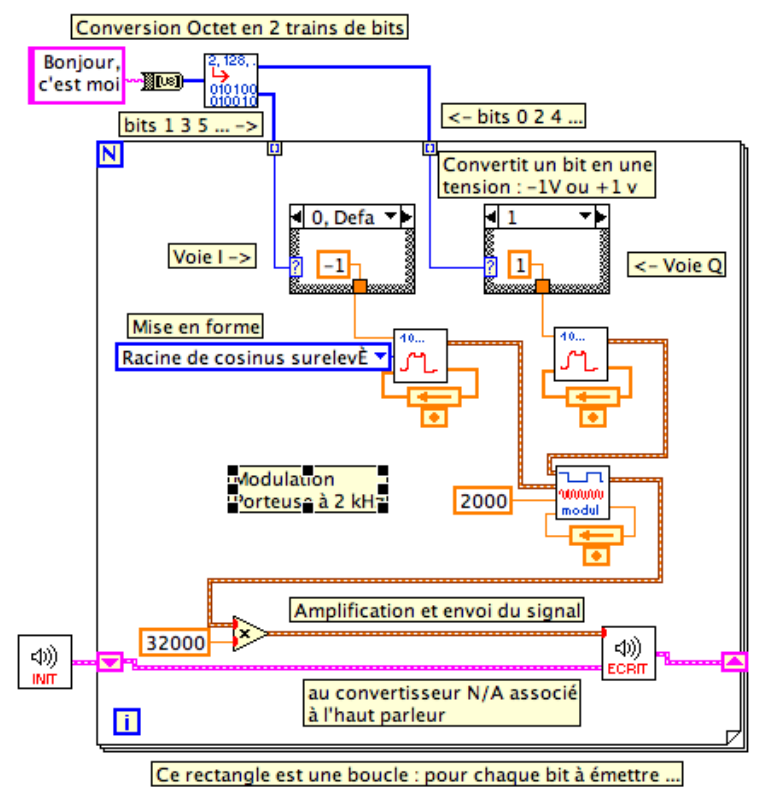

fig 3 : Diagramme de l'émetteur

Les étudiants construisent ce diagramme à partir du catalogue de boîtes qui leur sont fournies. Il doivent ainsi réfléchir sur l'ordre et le rôle de chaque boîte (le catalogue comprenant les boîtes dédiées à la réception).

Une fois le diagramme construit avec les paramètres par défaut, le logiciel émet un message de quelques secondes. Ce message, trop court pour être analysé (en temps réel) permet aux étudiants de faire le lien entre la 
taille du message (qu'ils augmentent) et la durée d'émission.

Le bloc de mise en forme permet de choisir à l'aide de la boîte défilante soit un filtre en racine de cosinus surélevé (permettant de limiter la bande passante utilisée par le signal), soit un filtre de type porte (consommant une bande passante importante). Il est assez facile pour l'étudiant de différencier ces deux types de filtre en écoutant le signal sortant du haut-parleur du PC : avec une porte, les transitions brutales ce traduisent par des craquements, avec une racine de cosinus surélevé, les transitions sont douce et beaucoup plus agréables à écouter. Il suffit ensuite de discuter avec les étudiants pour faire le lien entre ce qu'ils entendent et l'encombrement spectral.

Bien plus tard, dans le TP, les étudiants sont amenés à rajouter une séquence permettant de détecter le début du signal ainsi que des bits supplémentaires donnant le temps à l'émetteur de se caler sur la bonne fréquence. De même, les étudiants se contentent d'une modulation de type BPSK.

Il est également possible d'analyser la sortie à l'aide de graphe, de spectre, ... Néanmoins, cette analyse sera plutôt réalisée sur le récepteur.

\subsection{Démodulation (4 heures)}

Comme pour la modulation, les étudiants disposent de blocs pour réaliser la partie démodulation et doivent les assembler.

Dans un premier temps, le but est d'afficher la constellation du signal. Les algorithmes de synchronisation n'étant pas encore en place, les étudiants se contentent d'observer la trajectoire dans le plan (I, Q) sans échantillonnage (qui permettrai d'afficher les points associés aux bits reçus). Pour cela, ils utilisent un « graphe $\mathrm{XY}$ » où l'on connecte les signaux en phase (I) et en quadrature (Q).

Sur les cartes son que nous utilisons sur PC, nous avons constaté qu'un signal généré à une fréquence de $2 \mathrm{kHz}$ devenait, après son enregistrement à l'aide du microphone un signal à $1999,95 \mathrm{~Hz}$. Cet infime écart (25 ppm) suffit à provoquer une mauvaise réception (rotation dans la constellation). Nous indiquons donc aux étudiants, dans un premier temps d'utiliser 1999,95 $\mathrm{Hz}$ comme fréquence pour la réception.

\subsection{1 Étude de l'impact d'un déphasage}

Les émetteurs et récepteurs étant deux applications distinctes, elles ne sont pas démarrées simultanément et les cosinus et sinus utilisés pour la démodulation sont donc déphasés par rapport à ceux du modulateur. Cela ce traduit par un déphasage sur la constellation et sur les trajectoires (fig. 4). L'échantillonneur n'étant pas en place, il n'est pas possible d'obtenir une vraie constellation.

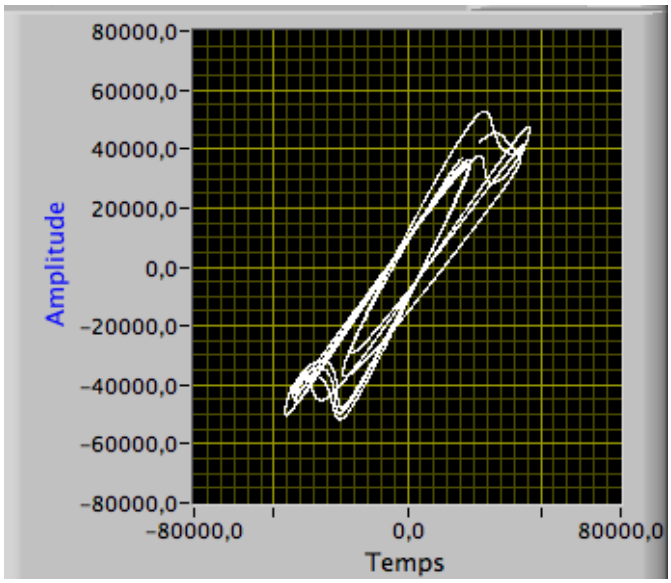

fig 4 : Trajectoire I, $Q$ sans compensation de phase

Les étudiants font plusieurs essais en lançant l'émetteur à différents instants (aléatoires) afin qu'ils comprennent que ce déphasage est aléatoire.

Après avoir fait le lien avec les équations vues cours, ils réfléchissent à la façon de corriger ce problème (estimation de l'angle et introduction d'un déphasage dans le modulateur). Ils mettent ensuite en oeuvre cette solution à l'aide d'un bloc permettant d'estimer la phase du signal.

Le lien avec la PLL est fait dans un autre cours dédiée, le but ici étant surtout de montrer les problèmes des télécommunications.

\subsection{2 Étude de l'impact d'un décalage de la fré- quence d'horloge}

Après avoir compensé le déphasage entre l'émetteur et le récepteur, les étudiants changent la fréquence d'horloge utilisée pour la démodulation dans le récepteur. Ils étudiants introduisent tout d'abord une erreur de $500 \mathrm{ppm}$ sur la fréquence du récepteur $(0,1 \mathrm{~Hz})$. Ils constatent que la trajectoire I,Q se met à tourner comme le montre la figure 5 (le logiciel fonctionnant en temps réel sur PC, les étudiants voient une animation).

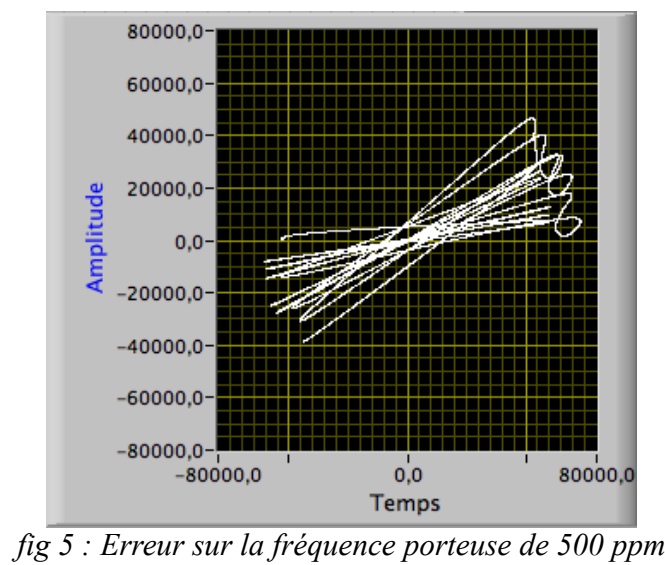


L'erreur sur la fréquence porteuse est encore accentuée : 5000 ppm $(1 \mathrm{~Hz})$ afin de montrer aux étudiants l'importance de la synchronisation (fig. 6).

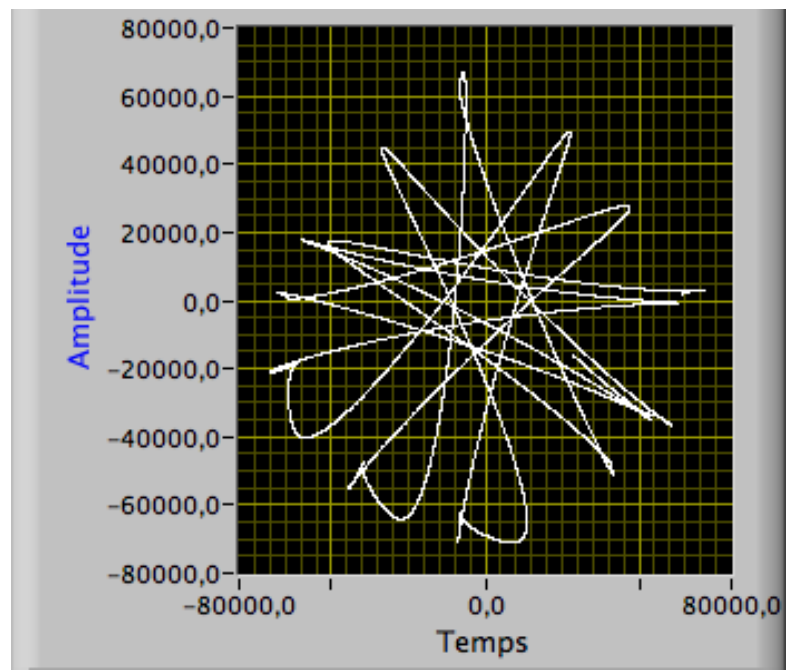

fig 6 : Erreur sur la fréquence porteuse de 5000 ppm

Une fois l'erreur constatée, les étudiants réfléchissent sur le lien entre la phase et la fréquence pour comprendre pourquoi la constellation tourne. Ils préconisent ensuite un moyen pour estimer l'écart de phase et utilisent le bloc dédié.

\section{3 Échantillonnage, Reconstruction du message}

\subsubsection{Synchronisation symbole}

Une fois le démodulateur achevé, le but est d'échantillonner le signal et de prendre la bonne décision. Pour cela les étudiants peuvent utiliser un bloc dédié.

En parallèle, les étudiants doivent visualiser le diagramme de l'oeil associé au canal I (fig. 7) et au canal Q.

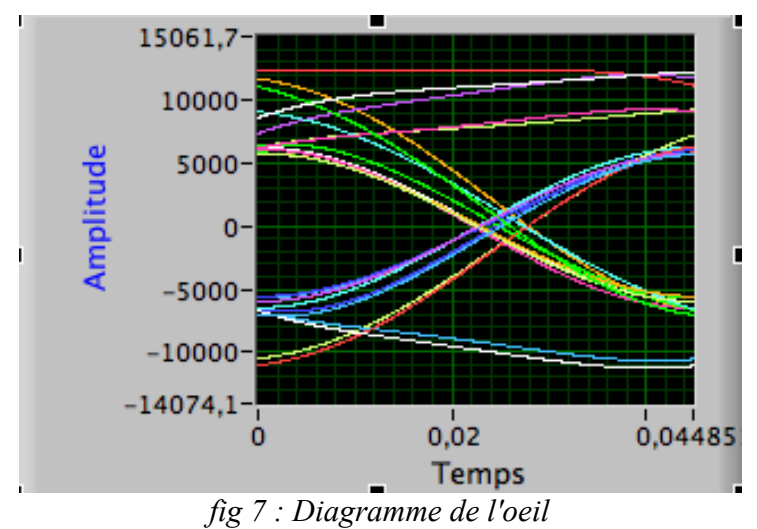

Les étudiants retrouvent des motifs qui leurs sont connus et presque aucun signal dans le diagramme de l'oeil associé à Q (BPSK). Malheureusement, ils se rendent compte en mettant en marche et arrêtant plusieurs fois l'émetteur qu'il y a un déphasage différent à chaque remise en marche de l'émetteur (non synchronisé avec le récepteur). Dès lors, l'instant optimum de décision varie. Si l'instant d'échantillonnage n'est pas ajusté, on peut être amené à prélever l'échantillon au milieu de la figure 7 par exemple.

Dès lors, ils sont amenés à étudier un algorithme de synchronisation symbole : l'early late gate synchronizer [3]. Cet algorithme illustré par la figure 8 prélève 3 échantillons proches : un échantillon associé à l'instant d'échantillonnage, un échantillon juste avant (Early) et un juste après (Late). On compare ensuite l'amplitude de la valeur absolue de l'échantillon E (early) et L (late) :

- si L>E (première ligne fig. 8), cela signifie qu'il faut augmenter décaler vers la droite l'instant d'échantillonnage,

- $\quad$ si $\mathrm{L}=\mathrm{E}$ (approximativement), cela signifie que l'on est à l'instant optimum,

- $\quad$ si $\mathrm{L}<\mathrm{E}$, cela signifie qu'il faut échantillonner plus tôt.

Cette étude ne ce fait évidement que lors d'une transition (état haut vers bas ou bas vers haut).

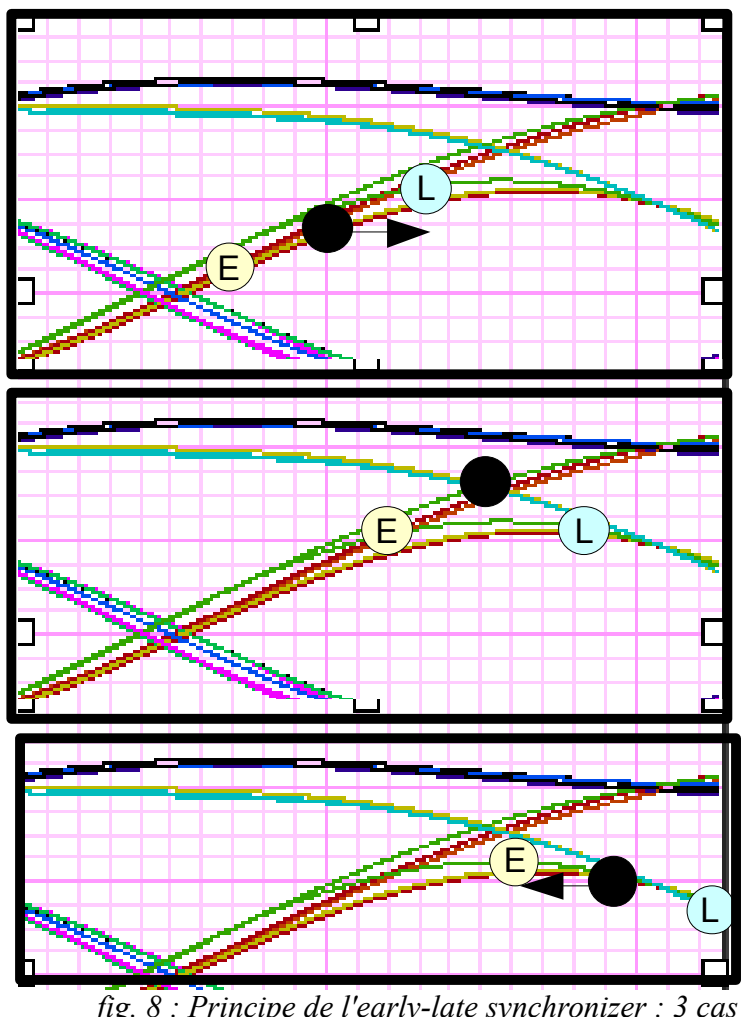

fig. 8: Principe de l'early-late synchronizer : 3 cas

Après avoir analysé cet algorithme, les étudiants utiliser le bloc de synchronisation fourni par l'enseignant. Ce bloc, en plus de synchroniser la phase, permet de montrer sur le diagramme l'évolution de l'instant d'échantillonnage à l'aide d'un curseur. Les étudiants prennent ainsi conscience du caractère adaptatif de cet algorithme. 


\subsubsection{Prise de décision et reconstruction du mes- sage}

La prise de décision est réalisée à l'aide du signe des échantillons prélevés étant donné qu'il n'y a que deux états.

Les étudiants disposent ensuite d'outils permettant d'assembler les bits pour les convertir en chaîne de caractère.

Un test du récepteur sans émission les convainc assez rapidement qu'il est nécessaire de rajouter une séquence permettant de marquer le début du message. La détection de cette séquence (à un une inversion de bit près) est réalisée par un bloc dédié.

À nouveau, le système ne fonctionne pas puisqu'il n'a pas le temps de corriger la phase et de trouver l'instant d'échantillonnage assez rapidement. C'est pourquoi les étudiants sont amenés à insérer une séquence donnant le temps au récepteur de ce synchroniser.

\section{RETOUR D'EXPÉRIENCE}

Cet ensemble de 3 TP a été utilisé pendant deux années en IUT Réseaux et Télécommunication à Roanne.

En cours, les étudiants voient souvent des diagrammes sans comprendre l'intégralité des éléments et surtout sans voir le lien entre eux (un signal en entrée, un signal en sortie). Les premiers TP forcent les étudiants à regarder le cours et à essayer de comprendre le rôle de chaque composant et surtout l'ordre dans lequel les placer.

Le choix de modulation BPSK peut paraître simpliste, mais permet aux étudiants de prendre conscience de leurs lacunes. Par ailleurs, le fait pour les étudiants d'entendre le signal modulé les motivent.

Dans la deuxième partie, les étudiants doivent retrouver à l'aide des formules de produit de cosinus l'effet d'un retard ou d'un déphasage. Alors qu'en cours ces l'utilisation de ces formules anesthésie les étudiants, ils les regardent avec un peu plus d'intérêt et de curiosité puisqu'ils doivent les comprendre pour répondre aux questions. Il est néanmoins nécessaire d'animer le TP et d'aider les étudiants les moins doués en mathématiques.

La troisième partie apparaît plus dure aux étudiants puisqu'il est nécessaire de rentrer plus dans la programmation LabView. La première année d'utilisation où l'algorithme de l'early late gate synchronizer était programmé directement par les étudiants. Seuls quelques étudiants étaient arrivés à le programmer. La deuxième année où le bloc était fourni, les étudiants se sont concentrés sur les aspects "télécom » et ont pu aller plus loin dans le TP. Cette dernière partie reste tout de même complexe pour les étudiants.

Cette année, ce TP a été transformé en TD sur machine afin de laisser des séances de TP libres pour l'étude de
l'OFDM et du CDMA (TP crées cette année). Étant donnée la durée d'un TD, les étudiants n'ont plus « câblés » l'émetteur récepteur, mais se sont limités à l'étude des différents signaux sur un émetteur récepteur fournis.

$\mathrm{Au}$ vu des résultats, il apparaît que les étudiants ont cherché à comprendre le rôle de chaque bloc individuellement (pour répondre aux questions). Néanmoins, comme ils n'ont pas eu la difficulté de devoir câbler le système complet, ils n'ont pas mémorisé dans quel ordre associer les blocs et leurs rôles.

\section{CONCLUSION}

Cette communication présente un exemple d'utilisation de labview ainsi que de la carte son pour réaliser un TP de communication numérique. Le but de ce TP est de montrer aux étudiants d'une manière didactique les problèmes de synchronisation et de reconstruction de message. L'utilisation de la BPSK rend assez intuitif certains points la correction de ces problèmes. L'utilisation de la bande audible permet aux étudiants d'écouter le signal et de mesurer l'importance des filtres de mise en forme. Il est également possible d'ajouter du bruit en soufflant sur le microphone, ...

Ce type de TP est surtout destiné à un public d'étudiant en IUT plus attiré par la pratique et plus réservé sur l'aspect mathématique.

De nombreuses perspectives existent : étude de QAM, ajout de filtres adaptatif type LMS pour réaliser de l'égalisation (en lien avec le module de traitement du signal). Néanmoins, elles se heurtent souvent au nombre assez limité de TP en télécom numérique (4).

\section{Bibliographie}

[1] GRESILOG S.A., "Boîte à outils communication pour Mustig", http://www.lis.inpg.fr/mustig.

[2] A. Layec, "Modnum Boîte à outils Scilab pour les systèmes de communication", http://www.scicos.org/ScicosModNum/modnum web/we b/fr/fr.htm.

[3] J.G. Proakis, "Digital Communications" 4ème édition, McGraw-Hill (2000), ISBN 978-0072321111. 